\title{
La Línea Negra como reconocimiento de territorios ancestrales de las comunidades indígenas de la Sierra Nevada de Santa Marta*
}

\author{
Leandro Alberto López Rozo**
}

Recibido: 20 de marzo de 2020 • Aprobado: 10 de mayo de 2020

\section{Resumen}

El respeto por los territorios ancestrales de las comunidades étnicas en Colombia ha representado un punto histórico de fricción social y violencia constante entre los distintos protagonistas de la violencia de nuestro país. El presente documento se próxima y aborda el plexo normativo y jurisprudencial relacionados con la protección de los espacios sagrados de los pueblos arhuaco, kogui, wiwa y kankuamo de la Sierra Nevada de Santa Marta, consignados o delimitados en lo que dichas comunidades étnicas han denominado como la "Línea Negra" (Séshizha, en la lengua kággaba del pueblo kogui; Shetana Zhiwa, en la lengua damana del pueblo wiwa; o Seykutukunumaku, en la lengua iku del pueblo kankuamo), concepto espiritual, cultural y físico de la Ley de Origen.

El reconocimiento del Estado colombiano a las comunidades indígenas de la Sierra Nevada de Santa Marta, de sus sitios sagrados y ancestrales a través de

\footnotetext{
El presente artículo es producto del proyecto de investigación titulado: "La delimitación de la Línea Negra o Sé Shiza de las comunidades indígenas de la Sierra Nevada de Santa Marta”, adelantado en el marco del programa de la Maestría en Derecho Administrativo de la Universidad Libre de Colombia, Sede Bogotá D. C.

Citar como: López Rozo, L. A. (2020). La Línea Negra como reconocimiento de territorios ancestrales de las comunidades indígenas de la Sierra Nevada de Santa Marta. Revista IUSTA, 53, 45-67. DoI: https://doi.org/10.15332/25005286.6271

*** Abogado egresado de la Universidad Católica de Colombia, con especializaciones en Derecho Privado Económico en la Universidad Nacional de Colombia, Derecho Probatorio en la Universidad Sergio Arboleda y Derecho Administrativo y Constitucional en su alma mater. Actualmente cursa tercer semestre de Maestría en Derecho Administrativo en la Universidad Libre de Colombia. Correo: lealloro@gmail.com. oRcid: 0000-0003-2284-0409.
} 
varias manifestaciones de las ramas de poder público —actos administrativos y sentencias-, constituye un gran paso para entender la importancia de las minorías étnicas que propende por el respeto y conservación de los recursos naturales y la relevancia tanto en sus vidas materiales como espirituales, por tal razón es apropiado estudiar si el andamiaje normativo es suficiente y adecuado para que se conserve y mantenga los sitios sagrados que delimitan la "Línea Negra" y que se encuentra en la memoria ancestral de las autoridades espirituales de nuestros hermanos mayores.

Palabras clave: Línea Negra, territorios ancestrales, Ley de Origen.

\title{
The "Black Line” (“La línea negra”) as Acknowledgement of the Ancestral Territories of the Indigenous Communities of the Sierra Nevada de Santa Marta
}

\begin{abstract}
Respect for the ancestral territories of the ethnic communities in Colombia has represented a historical point of social friction and constant violence between the different protagonists of violent conflicrt in our country. This text approaches and addresses the normative and jurisprudential plexus related to the protection of the sacred spaces of the Arhuaco, Kogui, Wiwa, and Kankuamo peoples of the Sierra Nevada de Santa Marta, set down or delimited in what these ethnic communities have called the "Black Line" (Séshizha, in the Kagaba language of the Kogui people; Shetana Zhiwa, in the Damana language of the Wiwa people; or Seykutukunumaku, in the Iku language of the Kankuamo people), a spiritual, cultural, and physical concept of the Law of Origin.

The recognition by the Colombian State of the indigenous communities of the Sierra Nevada de Santa Marta, and of their sacred and ancestral sites, through various actions of the branches of public power-administrative acts and court decisions-is a great step towards understanding the importance of ethnic minorities, advocating for the respect and conservation of natural resources and the relevance of both their material and spiritual lives. For this reason, it is appropriate to study whether the normative scaffolding is sufficient and adequate for the preservation and maintenance of the sacred sites that comprise the "Black Line", which is found in the ancestral memory of the spiritual authorities of our "elder brothers".
\end{abstract}

Keywords: Black Line, ancestral territories, Law of Origin. 


\section{A "LiNHA NEGRA" COMO RECONHECIMENTO DOS TERRITÓRIOS ANCESTRAIS DAS COMUNIDADES INDÍGENAS da Serra Nevada de Santa Marta}

\section{Resumo}

O respeito pelos territórios ancestrais das comunidades étnicas na Colômbia tem representado um ponto histórico de fricção social e de violência constante entre os diferentes protagonistas da violência no país. Este documento se aproxima da estrutura normativa e jurisprudencial relacionada com a proteção dos espaços sagrados dos povos Arhuaco, Kogui, Wiwa e Kankuamo da Serra Nevada de Santa Marta, consignados ou delimitados naquilo a que essas comunidades étnicas chamaram a "Linha negra" (Séshizha, na língua Kággaba do povo Kogui, Shetana Zhiwa, na língua Damana do povo Wiwa, ou Seykutukunumaku, na língua Ikudel do povo Kankuamo), um conceito espiritual, cultural e físico da Lei de Origem.

O reconhecimento pelo Estado Colombiano das comunidades indígenas da Serra Nevada de Santa Marta, dos seus locais sagrados e ancestrais através de diversas manifestações dos ramos do poder público - atos administrativos e julgamentos - é um grande passo para compreender a importância das minorias étnicas, que proponde pelo respeito e pela conservação dos recursos naturais, bem como pela relevância para a sua vida material e espiritual. Por essa razão, é conveniente estudar se a estrutura normativa é suficiente e adequada para preservar e manter os locais sagrados que delimitam a "Linha negra" e que se encontram na memória ancestral das autoridades espirituais dos nossos irmãos mais velhos.

Palavras-chave: Linha negra, territórios ancestrais, Lei de Origem.

\section{Introducción}

Como un prolegómeno del presente artículo, es el concepto de sacralidad o santidad de los lugares o sitios de las comunidades indígenas de la Sierra Nevada de Santa Marta, para ello se hace necesario indicar que la acepción de "sagrado" se asocia o se encuentra íntimamente ligado al concepto religioso, y en especial a la religión católica; luego es indispensable hacer alusión, como lo señala el Instituto Colombiano de Antropología e Historia Colombiano, a otras expresiones que permitan desmarcarse de la concepción antes dicha, esto es, referirse de los espacios como 
"sitios de origen", "sitios de gobierno", "sitios de poder", "sitios de respeto", "sitios prohibidos", "sitios de veda" o "sitios de encanto" (Saade y Páramo, 2018, p. 16). Aunado a lo anterior, la adecuada forma de referirse de los espacios ancestrales permitirá comprender en mejor forma la denominada la república unitaria y la democracia participativa y pluralista en que se funda nuestro Estado Social de Derecho, conforme lo establece el artículo $1^{\text {o }}$ de nuestra, ya mal trecha, Constitución Política de 1991.

La sacralidad es de valía para las comunidades indígenas de la Sierra Nevada de Santa Marta, los sitios de veda se encuentran en cabeza o en la memoria de determinadas personas de la comunidad que se entendería para nosotros, los hermanos menores — hombres blancos—, como sacerdotes de la sociedad, es decir, los sabedores de las enseñanzas ancestrales que para las comunidades en comento son los mamos. Son "religiosa" y políticamente figuras representativas y que pueden ser blanco de coacción o desaparición física de grupos armados al margen de la ley, con el firme propósito de controlar los territorios y una "profanación" que siembra el terror en la comunidad.

Es, pues, necesario señalar, como un norte del presente documento, que la comunidad jurídica comprenda la cosmovisión de las indicadas comunidades étnicas frente a la explotación de los recursos naturales y los efectos que ello significa, habida cuenta de que el Estado y los particulares deberán hacer uso del trámite administrativo de la consulta previa, con el fin de proteger este derecho fundamental de las poblaciones indígenas de la Sierra Nevada de Santa Marta; tema tan apropiado en medio de una pandemia mundial que ha puesto a reflexionar a la sociedades frente al gasto desenfrenado de los recursos no renovables.

El artículo contiene un alto grado de relevancia social, en la medida de que se analizará si el Estado colombiano tiene la capacidad de materializar la delimitación de los espacios sagrados de las plurimecionadas comunidades indígenas, en contraposición a la tensión que tiene que soportar el Gobierno Nacional de cara a la exploración y explotación de compañías nacionales y extranjeras de los recursos naturales en los sitios sagrados establecidos y reconocidos en la Línea Negra, respaldado por normas internacionales y nacionales.

Aunado a lo anterior, es palpable que el Estado, a través de las ramas del poder público - judicial y ejecutiva-, ha hecho ingentes esfuerzos para lograr el respeto a los lugares ancestrales de las comunidades indígenas de la Sierra Nevada de Santa Marta; circunstancia que no se observa con el resto de la población étnica 
del país, pues, a voces del Departamento Administrativo Nacional de Estadística —DANE-(Censo General), se registran 87 pueblos indígenas en todo el territorio nacional, sin que se evidencie una protección tan especial como la adelantada con las comunidades de la Sierra Nevada de Santa Marta.

\section{Los territorios ancestrales en Colombia}

Desde la mal llamada "Conquista" (siglo xvi, los reinos de España y Portugal), que en realidad se trató del etnocidio más vergonzante de la historia de la humanidad después del exterminio de los judíos en la Segunda Guerra Mundial — campos de concentración-y de la muerte en masa de la oposición en el régimen soviético de Iósif Stalin — gulags-, la población indígena ha soportado los actos más atroces de extinción, hasta nuestros tiempos, pues no ha sido otro fin - antes y ahora- que la exploración, explotación y extracción de los recursos naturales en territorios ancestrales por parte grandes compañías extrajeras respaldadas por Estados económicamente dominantes, y que se han denominado "instituciones económicas extractivas” (Acemoglu y Robinson, 2015), conforme lo han señalado destacados economistas estadounidenses.

Esa situación, inevitable para las poblaciones étnicas, ha generado circunstancias de gran tensión con el Estado y las empresas privadas, en territorios en que se ha desarrollado el conflicto armado interno Colombiano, lo que ha denominado la doctrina nacional como los “campos sociales minados" (Rodríguez, 2017), esto es, zonas que giran alrededor de las economías extractivas y que generan gran tensión de poder entre las empresas, la comunidades ubicadas en los territorios, grupos armados al margen de la ley (guerrillas, paramilitares, bandas criminales, disidencias de ex-FARC, entre otros grupos), y generan lugares apartados del país con gran impacto de violencia sobre la población; luego ha surgido como único remedio para zanjar los intereses contrapuestos el ejercicio del derecho fundamental a la consulta previa (Rodríguez, 2017), por parte de las comunidades indígenas, negras y gitanas o rom (grupos étnicos).

El territorio ${ }^{1}$ específico a que se hace alusión en el presente artículo corresponde a la Sierra Nevada de Santa Marta, constituido como el sistema montañoso más alto del mundo:

"Nuestro territorio. Los territorios que se encuentran en la Sierra Nevada de Santa Marta son nuestros, allí es donde tenemos escritas nuestras leyes la historia que sin ellas no seríamos pueblo de cultura 
[...] en un litoral con $5775 \mathrm{msnm}$; sus dos picos —Colón y Bolívar- son los más altos de Colombia, y tiene una extensión de $17.000 \mathrm{~km}^{2}$ sobre la costa del mar Caribe (Parques Nacionales Naturales de Colombia, 2010). Ubicada entre los departamentos de Magdalena, La Guajira y el Cesar, hace parte del parque nacional natural Sierra Nevada de Santa Marta (acuerdo 25/1977) y del parque nacional natural Tayrona (véase Viloria de la Hoz, 2005, julio), administrados ambos por el MAVDT [Ministerio de Ambiente, Vivienda y Desarrollo Territorial]. La tradición oral indígena de la Sierra reconoce cuatro grupos originarios del macizo: kogui, wiwa, kankuamo y arhuaco, cada uno con su propios territorio y lengua; pertenecientes todos a la familia lingüística chibcha y habitantes de la Sierra [...].

\section{La Sierra}

[...] es parte del territorio ancestral de los cuatro pueblos indígenas, demarcado por la línea negra o zonas teológicas, una serie de lugares ubicados usualmente en el nacimiento de los ríos o en montículos que referencian sitios sagrados (Observatorio de Derechos Humanos de la Vicepresidencia de la República, 2010). Sus límites tradicionales fueron reconocidos y registrados por el Estado colombiano en 1973, mediante resolución 002 del 4 de enero del Ministerio de Gobierno [...]. (Sierra, Castelblanco, Prada, Salinas y Lemaitre, 2010, p. 154)

Como "campo social minado" (Rodríguez, 2017), la Sierra Nevada de Santa Marta, por

[...] sus particularidades físicas y su condición de reserva natural han contribuido a la expansión de cultivos de uso ilícito y, paradójicamente, han dado vía libre a la destrucción de los ecosistemas [...], pues una de sus principales riquezas lo constituye [...] el agua, cuya producción se estima en 10 mil millones de metros cúbicos al año, aportados por sus diferentes ríos, aprovechados por la agricultura

diferente. Esta sierra nevada nos fue entregada a los cuatro grupos étnicos desde el principio del mundo, razón por la cual somos dueños. No como lo considera el gobierno colombiano un Parque Nacional y Patrimonio del resto de la Humanidad.

La Sierra Nevada de Santa Marta. Es la cuna de nuestros antepasados, Sherankua la creó para que las cuatro etnias viviéramos como hermanos en ella, cuidándola y no disgustándola ni atormentándole. Las cuatro étnicas de la sierra, son como cuatro hijos que tiene. Desde el principio los partió y les dio leyes, organización, lengua e historia para que cada una de ellas la cuidara y protegiera. Son indígenas que llevan parecida cultura, leyes, con distinta lengua y raíces semejantes" (Coronado, 2015). 
y el abastecimiento de acueductos de los centros urbanos, beneficiando a cerca de 1.5000 .000 (sic) habitantes. Debido a sus características geográficas y climáticas, y al asentamiento de pueblos indígenas, la Sierra representa un atractivo importante para los inversionistas interesados en el ecoturismo y el etnoturismo (Romero, 2006) [...]. (Becerra, 2009, p. 100)

El conflicto armado interno en territorios ancestrales ha significado, además de la desaparición "material de sus poblaciones", el

[...] deterioro de su tejido social y la transformación de sus expresiones culturales. Adicionalmente, la puja por el control territorial que ejercen grupos armados legales e ilegales ha ocasionado la invasión de sus resguardos, en desmedro de su ejercicio de gobierno propio y dando lugar a distintos tipos de vulneración de sus lugares sagrados. La violencia armada profana y corrompe la sacralidad de espacios que ordenan la vida y el territorio, mientras la construcción de instalaciones militares o policiales en dichos lugares viola prescripciones rituales sobre el modo de habitarlos o entrar en contacto con ellos, lo cual deriva en una serie de trastornos sociales y ecológicos mayúsculos. (Saade y Páramo, 2018, p. 43)

Corresponde, entonces, acometer someramente el análisis de las normas internacionales que abordan el reconocimiento y la protección de los territorios ancestrales. Una primera mirada recae sobre la Conferencia de Berlín (1884 y 1885), el denominado por los historiadores como el reparto de África (Ceamanos, 2016), este fue el acuerdo del abuso del eurocentrismo a través de las potencias mundiales de la época, sobre el continente africano y que fue, a la postre, la génesis de la mayoría de los conflictos armados que llegaron con posterioridad a dicho continente. Esa intromisión cultural, aunque no se crea, estableció un vestigio sobre la contribución al bienestar y el desarrollo de las poblaciones indígenas en las colonias y territorios ocupados.

Es importante destacar, además, que en el Tratado de Paz firmado en Versalles (1919), en su acápite sobre el Pacto de la Sociedad de Nacionales (art. 22) estableció "[...] la tutela [...] sobre los territorios habitados por pueblos aún no capacitados para dirigirse por sí mismo en las condiciones particularmente difíciles del mundo moderno [...]" (Natan, 2017, p. 73). En conexidad con lo anterior, tenemos el 
[...] parágrafo I de la Recomendación sobre la reglamentación del trabajo forzoso, 1930 (núm. 36), que dice así: [...] todas las leyes o reglamentos concernientes a la indemnización en caso de accidente, enfermedad o muerte del trabajador, deberían ser impresos por las autoridades competentes en una o varias lenguas indígenas, de suerte que los trabajadores interesados y la población en al cual son reclutados puedan conocer el contenido de estos textos [...]. (Natan, 2017, p. 75)

En 1957, la Organización Internacional del Trabajo ${ }^{2}$ adoptó el Convenio No. 107 “[...] relativo a la protección e integración de las poblaciones indígenas y de otras poblaciones tribales y semitribales [...]”; en dicho instrumento internacional se encuentra, aunque muy tenue, el reconocimiento a las comunidades indígenas sobre "[...] las tierras tradicionalmente ocupadas por ellas" (art. 11), así como la prohibición de trasladar la población, salvo por “[...] razones previstas por la legislación nacional relativas a la seguridad nacional, al desarrollo económico del país o a la salud [...]" de las misma comunidad asentada (art. 12). Este convenio es bastamente criticado, al considerarse que se expidió o se aprobó en

[...] una época donde los pueblos indígenas y tribales eran considerados como sociedades "atrasadas" y se creía fervientemente que para que pudiesen sobrevivir necesitaban integrarse a la sociedad común y someterse a sus reglas. Sin embargo, no se debe dejar de lado que a diferencia del indigenismo segregacionista colonial, o el segregacionismo asimilista republicano, el integracionista valoró al indígena en sí reconociendo su complejidad socio-cultural y su situación especialmente vulnerable frente a los desafíos de la sociedad progresista y moderna. Es decir, el principio de integración nacerá con la intención de rescatar los valores, la personalidad histórica y cultura de los pueblos indígenas, pero buscando que los mismos lograsen alcanzar la asimilación a la colectividad nacional incorporando a sus vidas las técnicas modernas. (Víquez, 2019, p. 895)

En 1989, la misma organización internacional expidió el Convenio n. ${ }^{\circ}$ 169, sobre pueblos indígenas y tribales en países independientes, documento trascendental

2 La Organización Internacional del Trabajo, expidió los siguientes instrumentos internacionales a favor de las comunidades indígenas: 1) el Convenio sobre el reclutamiento del trabajadores indígenas (1936); 2) el Convenio sobre los contratos de trabajo (1939);3) el Convenio sobre sanciones penales (1936); 4) la recomendación sobre la duración máxima de los contratos escritos de los trabajadores indígenas (1939); 5) la recomendación sobre la inspección del trabajo (1939); y 6) el Convenio sobre los contratos de trabajo (1947). 
para el amparo de los derechos de los grupos étnicos en sus usos y costumbres, pero además de gran importancia a la protección de las tierras ancestrales, para ello estableció un capítulo denominado tierras (Parte II, art. 13). En dicho aparte del instrumento internacional, establece que los gobiernos que ratifiquen el convenio,

[...] deberán respetar la importancia especial que para las culturas y valores espirituales de los pueblos interesados reviste su relación con las tierras o territorios, o con ambos, según los casos, que ocupan o utilizan de laguna otra manera, y en particular los aspectos colectivos de esa relación.

El Congreso de la República de Colombia, mediante a la Ley 21 de 1991, aprobó dicho convenio. A diferencia del Convenio 107, el 169

[...] se ubica en un sitio importante en cuento al reconocimiento de los valores culturales y espirituales que los pueblos indígenas atribuyen a sus territorios ancestrales, dado que son esenciales para garantizar el conjunto más amplio de sus derechos. Al respecto, un elemento sustancial que contiene el Convenio 169 es el reconocimiento del concepto de territorio como la totalidad del hábitat que los pueblos ocupan o utilizan conforme a su visión y cultura. Con base en ese concepto, se han logrado cambios sustanciales en la aplicación del derecho internacional e interamericano de los derechos humanos, así como es las normas de los países que han ratificado el Convenio. (Vallejo, 2018, p. 105)

Para tratadistas vernáculos, el Convenio 169 de 1989 es una norma internacional que establece un estructura más amplia e incluyente que la Constitución Política de 1991, pues

[...] se inicia por una especie de admonición sobre la importancia que para estos pueblos tienen la tierra y los territorios, asumidos caso como sinónimos (art. 13). Posteriormente, en el artículo 14 se precisa que ese reconocimiento de propiedad y posesión debe extenderse a las tierras que tradicionalmente ocupan. A ello habría que agregar los derechos que les correspondan sobre tierras a las que, aun cuando ocupadas permanentemente, hayan tenido acceso para sus actividades tradicionales y de subsistencia (resaltando especialmente el caso de pueblos nómadas o agricultores itinerantes). [...] Igualmente, los pueblos mencionados tienen derecho a no ser trasladados de las tierras y territorios que ocupan. No obstante, si por alguna 
situación el traslado es necesario, solo puede proceder si se ha realizado un proceso de consulta previa informada y se ha otorgado el consentimiento para ello, garantizándole a su vez las indemnizaciones que ello genere, manteniendo en todo caso el derecho a retornar a los territorios cuando desaparezcan las causas que motivaron el traslado (art. 17). (Borrero, 2018, p. 30)

Aunado a lo anterior, tenemos como instrumentos internacionales la Carta de las Naciones Unidas y la Declaración sobre la concesión de la independencia a los países y pueblos coloniales, la cual fue aprobada por la Asamblea General en la resolución 1514 del 14 de diciembre de 1960; así como la Declaración Universal de Derechos Humanos —arts. 7 y 17-, la Convención para la Prevención y la Sanción del Delito de Genocidio (1948) y la Convención Internacional para la Eliminación de todas las formas de Discriminación Racial (1960), el Pacto Internacional de Derechos Civiles — art. 27-y la Convención sobre los Derechos del Niño33.

Para nuestro ámbito nacional, la Constitución Política de 1991 estableció un marco constitucional proteccionista de la población étnica colombiana (indígenas, negritudes y rom), en lo que respecta a los derechos en territorios de las comunidades indígenas encontramos los arts. 286 (reconocimiento de territorios indígenas), 321 (la posibilidad de crear provincias con territorios indígenas), 329 (conformación de las entidades territorios indígenas), 330 (la gobernabilidad de la los territorios indígenas) y 356 (beneficios económicos de las entidades territoriales indígenas).

De cara al plexo normativo colombiano se tiene la Ley 160 de 1994 "por la cual se crea el Sistema Nacional de Reforma Agraria y Desarrollo Rural Campesino [...]”; Decreto 2164 de 1995, "por el cual se reglamenta parcialmente el Capítulo XIV de la Ley 160 de 1994”; Decreto 1396 de 1996, "por el cual se crea la Comisión Nacional de Territorios Indígenas y se crea el programa especial de atención a Pueblos Indígenas”; Decreto 1397 de 1996, "por el cual se crea la Comisión Nacional de Territorios Indígenas y la mesa Permanente de Concertación con los pueblos y las organizaciones indígenas y se dictan otras disposiciones"; y, Decreto 1320 de 1998, "reglamenta la Consulta Previa con las comunidades indígenas y negras para la explotación de recursos naturales dentro de su territorio".

En igual forma la jurisprudencia constitucional colombiana ha hecho énfasis en el resguardo de sus derechos frente a su territorio (T-009/13 y T-693/11), ello en concordancia con la Ley 165 de 1994, norma que aprobó el Convenio sobre

Aprobado mediante la Ley 12 de 1991 por el Congreso de la República de Colombia. 
la Diversidad Biológica de 1992 y que reconoce "[...] la estrecha y tradicional dependencia de muchas comunidades locales y poblaciones indígenas que tienen sistemas de vida tradicionales basados en los recursos biológicos"; dicha norma establece en su artículo $8^{\circ}$ que

Con arreglo a su legislación nacional, respetará, preservará y mantendrá los conocimientos, las innovaciones y las prácticas de las comunidades indígenas y locales que entrañen estilos tradicionales de vida pertinentes para la conservación y la utilización sostenible de la diversidad biológica y promoverá su aplicación más amplia, con la aprobación y la participación de quienes posean esos conocimientos, innovaciones y prácticas.

\section{La Línea Negra}

Atado al derecho a la tierra que tienen los pueblos indígenas arhuaco, kogui, wiwa y kankuamo de la Sierra Nevada de Santa Marta, la cosmovisión de los pueblos étnicos difiere en su totalidad frente a la visión del mundo que posee la cultura "civilizada", esto es, desde de los conceptos de vida, comunidad, naturaleza y territorio, hasta la misma creación de la tierra, como muy bien se puede extraer de la "Ley de Origen". Entonces, al existir una profunda relación tradicional y ancestral, con estribo en un concepto que interacciona entre lo ambiental, cultural y espiritual de los diferentes espacios sagrados, surge la denomina "Línea Negra" o red de conectividad y relaciones que integran su territorio a los principios de la vida, el planeta y el universo, que por supuesto puede extenderse a territorios que no se encuentran en el resguardo indígena (Molina, 2016).

Como antecedente de la denominada Línea Negra o Sé Shiza se encuentra la Resolución n. 02 de 1973 "[p]or la cual se demarca la Línea Negra o Zona Teológica de las comunidades indígenas de la Sierra Nevada de Santa Marta"; en dicho acto administrativo se señaló de manera simbólica la Línea Negra como aquella "área circular delimitada por accidente geográfico".

Para 1974 el Instituto Colombiano de Reforma Agraria (Incora), creó la reserva arhuaca 
[...] sobre los corregimientos de Azúcar Buena, Sabana Crespo y la zona centro occidental del corregimiento de Guatapurí, en los municipios de Pueblo Bello y Valledupar, departamento del Cesar (Gobernación del Cesar, 1999). Años después (en 1983), el Incora constituyó legalmente el resguardo Arhuaco sobre esta reserva y le anexó 10.900 ha para 195.900 (acuerdo 083/1983 y resolución 078/1983). En 1980, el turno fue para el pueblo kogui. El Estado colombiano destinó una extensión de 26.214 ha para la constitución de resguardo kogui-malayo, en la zona centro oriental de Valledupar, corregimiento de Chemesquemena, área de Guatapurí, y el corregimiento de Atanques (resolución 0109/1980 y acuerdo 109/1980). En 1995, la resolución 0837 del Ministerio del Interior reformó la resolución 002/1973 que reconoció legalmente la línea negra, "para concluir la ubicación de 54 sitios sagrados para los pueblos de la Sierra Nevada”. Finalmente, en abril del 2003, el Incora constituyó el resguardo Kankuamo con una extensión de 24.212,2 ha en la misma Sierra (Observatorio de Derechos Humanos de la Vicepresidencia de la República, 2010). (Sierra, Castelblanco, Prada, Salinas y Lemaitre, 2010, p. 155)

Posteriormente, los sitios y territorios ancestrales se materializaron en el Decreto 1500 de 2018,

Por el cual se redefine el territorio ancestral de los pueblos Arhuaco, Kogui, Wiwa y Kankuamo de la Sierra Nevada de Santa Marta, expresado en el sistema de espacios sagrados de la "Línea Negra”, como ámbito tradicional, de especial protección, valor espiritual, cultural y ambiental, conforme los principios y fundamentos de la Ley de Origen, y la Ley 21 de 1991, y se dictan otras disposiciones.

Lo anterior obedece, como ha sido su constante, a una deuda histórica con las comunidades indígenas, pues el Estado ha tenido, como lo señala la doctrina vernácula, acciones negativas para adelantar la identificación de los territorios (Albán, 2016), lo que a la postre no ha permitido allanar el camino para la reglamentación, mediante una ley estatutaria, del derecho fundamental a la consulta previa.

Determinar los sitios y lugares sagrados por fuera del resguardo indígenas implica, en la práctica, una reorganización del territorio de la comunidad étnica y de los entes territoriales que rodean el cabildo indígena, y los lugares que se establezcan como sagrados en la fijación de la línea negra por parte del Gobierno Nacional. En efecto, la determinación de estos nuevos territorios como sagrados redefine los 
territorios indígenas de las comunidades arhuaco, kogui, wiwa y kankuamo de la Sierra Nevada de Santa Marta del departamento del Magdalena, lo que conlleva unos efectos jurídicos.

La primera norma de que se tenga memoria frente a las comunidades indígenas y en nuestra reciente república hace alusión a la Ley 89 de 1890, "por la cual determina la manera como deben ser gobernados los salvajes que vayan reduciéndose a la vida civilizada", con la clara salvedad de la inconstitucionalidad de los artículos 1 , 5, 40 (C-139/96) y 11 (C-463/14); dicha disposición, por retrógrada y retardataria que sea, se estableció la organización de los cabildos indígenas y la independencia en sus normas internas, es decir surgió, a la postre, un reconocimiento tácito del Estado a la coexistencia y el desarrollo que tienen las poblaciones indígenas de autogobernarse, que para la Constitución Política de 1991 corresponda el desarrollo del principio constitucional de la diversidad étnica y cultural de la Nación. Postulado que se extraña en la Constitución de 1886.

Con la Constitución Ecológica de 1991 se dio una verdadera importancia a la población étnica y a los recursos naturales, además el Estado reconoce y protege la coexistencia de plexos normativos que rigen a los pueblos indígenas y el reconocimiento como entidad de derecho público especial de los cabildos y de las autoridades tradicionales; efectivamente se puede encontrar dichos principios en los artículos $1^{\circ}$ (Estado social de derecho), $7^{\circ}$ y $8^{\circ}$ (la diversidad étnica y cultural de la nación), 70 (la cultura como fundamento de la nacionalidad), 79 (el deber, del Estado de proteger la diversidad e integridad del ambiente y la conservación de las áreas de especial importancia ecológica), 286 (los cabildos como entes territoriales) y 330 (la autogobernación de los pueblos indígenas).

Concomitante con el advenimiento de la Constitución Política de 1991, el Congreso de la República expidió la Ley 21 de 1991, por medio del cual aprobó la suscripción del Convenio 169 de 1989, instrumento internacional "sobre Pueblos Indígenas y Tribales en Países Independientes", instrumento internacional que reconoce los derechos humanos de los pueblos indígenas, razón por la cual hace parte del Bloque de Constitucionalidad.

Dicha norma estableció en su artículo 13 que

[...] los gobiernos deberán respetar la importancia especial que para las culturas y valores espirituales de los pueblos interesados reviste su relación con las tierras 
o territorios, o con ambos, según los casos, que ocupan o utilizan de alguna otra manera, y en particular los aspectos colectivos de esa relación.

Subsecuentemente señala en el artículo 14 que

[...] en los casos apropiados, deberán tomarse medidas para salvaguardar el derecho de los pueblos interesados a utilizar tierra que no estén exclusivamente ocupadas por ellos, pero a las que hayan tenido tradicionalmente acceso para sus actividades tradicionales y de subsistencia [...]

Paralelamente, surge el Decreto-Ley 4633 de 2011 "por medio del cual se dictan medidas de asistencia, atención, reparación integral y de restitución de derechos territoriales a las víctimas pertenecientes a los pueblos y comunidades indígenas" y la Sentencia T-693 de 2011, en donde la Corte Constitucional puntualizó que "[c]on relación al derecho a la protección de las áreas sagradas o de especial importancia ritual y cultural, incluso si están ubicadas fuera de los resguardos, se observa que el Convenio 169 acoge un concepto amplio de territorio, al indicar que se consideran como tal, aquellas áreas de una comunidad que comprenden, no sólo las tituladas o habitadas, sino también aquellas que constituyen el ámbito tradicional de sus actividades tradicionales, sagradas o espirituales [...]", y subsecuentemente indicó que "[...] bajo este entendido, el territorio viene a ser el lugar donde las comunidades indígenas pueden desenvolverse según su cultura, su saber y sus costumbres. Es decir, un espacio físico bajo la influencia cultural y control político de sus propias costumbres".

Lo anterior es trascendental para entender la existencia de sitios sagrados de las comunidades indígenas por fuera de los territorios de los resguardos o cabildos, y que constituyen sitios sagrados que conectan a las autoridades ancestrales con los sentimientos espirituales con la tierra y la vida dentro de su cosmovisión.

Con posterioridad, el indicado estrado judicial, mediante la Sentencia T-009 de 2013, indicó que

(i) El derecho a la construcción de resguardos en territorios que las comunidades indígenas han ocupado tradicionalmente; (ii) El derecho a la protección de las áreas sagradas o de especial importancia ritual y cultural, incluso si están ubicadas fuera de los resguardos; (iii) E1 derecho a disponer y administrar sus territorios; (iv) El derecho a participar en la utilización, explotación y conservación de los 
recursos naturales renovables existentes en el territorio; (v) el derecho a la protección de la áreas de importancia ecológica; y (vi) el derecho a ejercer la autodeterminación y autogobierno. (Sentencia T-236 de 2012)

Para los pueblos arhuaco, kogui, wiwa y kankuamo de la Sierra Nevada de Santa Marta, es claro que existen territorios tradicionales y ancestrales, que se materializan en la red interconectada de lugares y recursos sagrados de lo que esas culturas indígenas han denominado la Línea Negra, que a la postre constituye, desde la cosmovisión de los indicados pueblos étnicos, su ámbito cultural y ambiental, pero ante todo la articulación de la materialización de las funciones de las autoridades públicas indígenas (Barragán, 2016).

Sobre el particular, en 1973 el Ministerio de Gobierno expidió la Resolución n. ${ }^{\circ}$ 22, "por la cual se demarca la Línea Negra o Zona Teológica de las comunidades indígenas de la Sierra Nevada de Santa Marta”. En dicho acto administrativo, de manera tibia, por no decir temerosa, se identificaron o demarcaron algunos puntos geográficos y se permitió el derecho al acceso a los mismos, así ordenó a los propietarios en donde aquellos estén ubicados con el fin de permitir las prácticas culturales dentro de sus territorios.

Con posterioridad el Gobierno Nacional, mediante la Resolución n. 837 de 1995 expedida por el Ministerio del Interior, reconoció la Línea Negra y la definió como la "[...] delimitación espiritual, dinámica y holística del territorio [...]" de los pueblos indígenas de la Sierra Nevada de Santa Marta, que además representa un sistema de espacios sagrados o tejido del territorio ancestral. Así mismo, se modificó la Resolución 000002 de 1973.

Frente a la débil normatividad que regulaba la Línea Negra de los pueblos indígenas de la Sierra Nevada de Santa Marta, los mismos dirigentes de las comunidades expresaron las imprecisiones en la identificación y la expresión de los "códigos ancestrales" contenidos en los actos administrativos mencionados. Dichas comunidades expresaron, además, la omisión de incluir los principios de la Ley de Origen, que a la postre detalla los espacios sagrados que la integran, generando un tejido de interconexión de tierra, litoral y mar. Igualmente, señalaron que tales actos limitan la gobernanza sobre los territorios que cubren la Línea Negra.

En torno a la jurisprudencia de la Corte Constitucional, el concepto de la Línea Negra fue abordado de manera somera, por no decir displicente, en la Sentencia T-634/99. En dicho expediente, la Procuraduría Delegada para Asuntos Étnicos 
promovió la acción constitucional en contra de la Gobernación del departamento de Cesar, el registrador nacional del Estado Civil y el registrador departamental del indicado ente territorial. Dicha delegada del Ministerio Público buscaba el amparo de los derechos fundamentales del pueblo arhuaco, toda vez que dicha comunidad indígena no había sido tenida en cuenta en la creación del municipio de Pueblo Bello, Cesar, y donde estaba conformada por unos corregimientos y veredas que se encontraban dentro de la Línea Negra; no obstante, de manera simplista dicho estrado judicial negó el amparo, ya que en su sentir era improcedente, en vista de que el Ministerio Público tenía en sus manos la interposición de la acción popular, pues se encontraba frente a derechos colectivos, argumento despachado en dos párrafos (Garzón, 2013).

Con posterioridad, en el fallo T-547 de 2010, el máximo órgano de cierre constitucional estudió la solicitud de amparo de varios accionantes representantes de los pueblos indígenas de la Sierra Nevada de Santa Marta, en atención a la expedición de la Resolución n. ${ }^{\circ} 1298$ de 30 de junio de 2006, mediante el cual el Ministerio de Ambiente, Vivienda y Desarrollo Territorial otorgó licencia ambiental a la empresa Brisa S. A., para que se adelantara un proyecto que se denominó "Construcción y Operación de la Fase 1 del 'Puerto Multipropósito de Brisa”, en el municipio de Dibulla en el departamento de La Guajira, la cual, según las comunidades indígenas, constituía territorios ancestrales y parte de la Línea Negra. En esta ocasión, nuevamente la Corte Constitucional hace referencia a las normas que gobiernan la Línea Negra pero no profundiza en el tema; sin embargo, ordenó a la sociedad suspender las actividades de desarrollo del proyecto y a las entidades involucradas adelantar el proceso de consulta (Guarín y Aldana, 2016).

Dentro del marco del cumplimiento de la Sentencia T-547 de 2010, la Corte Constitucional profirió el Auto n. ${ }^{\circ} 189$ de 2013, por el cual puntualizó que si bien las resoluciones 00002 de 1973 y 837 de 1995 expresan el reconocimiento jurídico que el Estado ha dado a la relación especial y espiritual que los cuatro pueblos de la Sierra Nevada de Santa Marta mantienen con su territorio ancestral, estas "[...] ya muestran su insuficiencia, requiriéndose disposiciones más ajustadas a las necesidades del colectivo indígena [...]”, razón por la cual instó al Gobierno Nacional, a través de las dependencias competentes, a que iniciaran las actividades tendientes a revisar, modificar, derogar o adicionar los indicados actos administrativos.

Un año posterior al indicado auto de la Corte Constitucional, la Sección Quinta de la Sala de lo Contencioso Administrativo Consejo de Estado, en fallo del 20 
de 2014, exp. n.o 2013-00008-01, modificó y amparó los derechos deprecados por la Fundación Misión Colombia (consulta previa sobre la diversidad étnica, social, cultural y religiosa, a la autonomía y al debido proceso) como agente oficioso de las comunidades indígenas de la Sierra Nevada de Santa Marta, habida cuenta de que en el trámite de expedición de la licencia ambiental por parte del Ministerio del Medio Ambiente, al proyecto ecoturístico Los Ciruelos, no se realizó la consulta previa, como lo ordena el Convenio 168 de la Organización Internacional del Trabajo.

En dicha providencia, el máximo órgano de cierre de la justicia contenciosa administrativa puntualizó que

La "Línea Negra", como concepción radial y perimetral del territorio indígena de los pueblos de la Sierra Nevada de Santa Marta, corresponde a dos modelos de categorías diferentes. La primera relacionada con la cosmovisión indígena de delimitación espiritual, dinámica y holística del territorio. La segunda tiene que ver con una concepción del área geométrica y estática occidental para definir un territorio. Respeto de ambas concepciones se prevé una forma de articulación intercultural a efectos de lograr no solo la protección y el respeto a las prácticas tradicionales culturales indígenas, sino la garantizar una relación intercultural funcional con la autonomía política y cultural, de la cual gozan los indígenas de la Sierra Nevada de Santa Marta. Así, no cabe duda de la importancia que tiene la "Línea Negra" para las comunidades indígenas, pues esta constituye una "articulación intercultural entre dos modelos diferentes de regulación territorial, a la vez que facilita la protección y el respeto por los territorios sagrados, así como de la riqueza cultural de los pueblos que la habitan y usan tradicionalmente”.

En el segundo gobierno del presidente Juan Manuel Santos, el ejecutivo se comprometió en el proceso de consulta previa del Plan Nacional de Desarrollo 2014-2018, por medio del cual se adelantarían las modificaciones a las resoluciones vigentes que gobernaban la Línea Negra, abrigando la nueva normatividad la integridad territorial y el desarrollo normativo concordante con la Ley 21 de 1991.

En el mismo año que iniciaba el indicado plan de gobierno, la Corte Constitucional, en una sentencia relevante señaló que la Línea Negra "es una zona de especial protección, debido al valor espiritual y cultural que tiene para los cuatro pueblos indígenas de la Sierra Nevada de Santa Marta" y que, en tal sentido, 
[...] el compromiso asumido por el Estado colombiano no se limita a la garantía de protección de algunos sitios al interior de la denominada línea negra, sino a la totalidad del territorio que incorpora la misma toda vez que corresponde a un espacio geo-referencial delimitado por un polígono que recrea un espacio determinado y no un conjunto de lugares sin conexión alguna en lugares aislados.

Por su parte, el Ministerio del Medio Ambiente, mediante la Resolución n. 0621 de 2002, se dispuso incorporar a los procesos de planificación y gestión ambiental de dicho ministerio, de la Unidad Administrativa Especial de Parques Nacionales Naturales y de las tres corporaciones autónomas regionales con jurisdicción en el territorio ancestral de los pueblos arhuaco, kogui, wiwa y kankuamo; los acuerdos adelantados con el Consejo Territorial de Cabildos en el marco de la elaboración del Plan de Desarrollo Sostenible de la Sierra Nevada de Santa Marta y aquellos futuros relativos a la implementación de los aspectos antes mencionados.

En ejercicio del derecho a la libre determinación, los pueblos indígenas arhuaco, kogui, wiwa y kankuamo de la mano con el Gobierno Nacional, y en cumplimiento del Auto n. 189 de 2013, elaboraron el 9 de diciembre de 2015, el denominado "Documento Madre - Jaba Seshizha”, el cual tuvo como propósito “[...] construir un puente de entendimiento y compresión entre el mundo de pensamiento indígena de la Sierra Nevada de Santa Marta y la Institucionalidad del Estado Colombiano para garantizar la protección [...]" del territorio ancestral. En dicho documento se acogieron los principios y fundamentos ancestrales de la Ley de Origen que edifica la integridad y conectividad material, espiritual, visible y no visible, en los territorios ancestrales que se materializa en la Línea Negra (Rodríguez, 2016).

Finalmente, el Gobierno Nacional expidió el Decreto n. ${ }^{\circ} 1500$ del 6 de agosto de 2018,

Por el cual se redefine el territorio ancestral de los pueblos Arhuaco, Kogui, Wiwa y Kankuamo de la Sierra Nevada de Santa Marta, expresado en el sistema de espacios sagrados de la Línea Negra, como ámbito tradicional, de especial protección, valor espiritual, cultural y ambiental, conforme los principios y fundamentos de la Ley de Origen, y la Ley 21 de 1991, y se dictan otras disposiciones.

Los instrumentos internacionales y los derechos convencionales allanan el camino para el cumplimiento a la delimitación de la Línea Negra o Sé Shiza como 
espacio sagrado de las comunidades indígenas arhuaco, kogui, wiwa y kankuamo de la Sierra Nevada de Santa Marta en el departamento de Magdalena, ello en concordancia con el plexo normativo y constitucional colombiano (Ballesteros, 2015).

La protección de la cosmovisión y la espiritualidad de una población étnica minoritaria en una república pluralista representa los valores y fines constitucionales que busca la Constitución Política de Colombia de 1991, además del respeto de los recursos naturales por una exploración y explotación con responsabilidad sostenible (Patarroyo y Benavides, 2014). El Estado ha establecido una serie de normas que permiten cumplir la delimitación de la Línea Negra o Sé Shiza, junto con una serie de pronunciamientos de las altas cortes que permiten acudir a la justicia colombiana y un procedimiento administrativo que blinda el cumplimiento del derecho fundamental de la consulta previa para el mismo Estado y los particulares (Rodríguez, 2012). Dicho instrumento

[...] es la herramienta para facilitar la concertación entre los pueblos indígenas y las partes que quieran intervenir el territorio indígena. Sin ella no hay espíritu de tolerancia, ni interés alguno en la situación de los pueblos indígenas, es deber de los gobiernos garantizar que espacios como la consulta previa, se realicen siempre a cabalidad, antes de que se expida cualquier autorización para desarrollar proyectos en territorios de circunscripción especial indígena. (Murillo y Duván, 2013, p. 100)

\section{Conclusiones}

Las comunidades indígenas de la Sierra Nevada de Santa Marta han logrado, tras 528 años de sometimiento de otras culturas y guerras recicladas en la historia de nuestro país, el reconocimiento de sus sitios o lugares ancestrales a través de actos administrativos y decisiones judiciales, circunstancia que materializa el preámbulo y los principios fundamentales de la Constitución Política de 1991, esto es, el fortalecimiento de la unidad nacional, el respeto a la autonomía de las entidades territoriales, la consolidación de un Estado pluralista y la efectividad de los derechos consagrados en la misma Constitución.

Entender la cosmovisión de las distintas poblaciones étnicas de la Sierra Nevada de Santa Marta constituye la reivindicación de años de lucha por un lugar en la sociedad colombiana, pero ante todo el reconocimiento de la Línea Negra como 
un concepto espiritual, cultural y físico de la Ley de Origen, principal norma de la creación de la tierra, el aire y el agua. Es, entonces, el reflejo de la aceptación del Estado de la existencia de otras formas observar los recursos naturales y de la espiritualidad que representa para ciertos grupos indígenas.

La consulta previa se constituye en la principal herramienta jurídica que tienen los grupos étnicos para proteger sus territorios sagrados y ancestrales ante los hermanos menores - hombres blancos-, de cara a los proyectos exploratorios y de explotación que proyecten las distintas entidades estatales con los particulares.

\section{Referencias}

Abello, C. J. (2012). Consulta previa en cosas de minería para comunidades indigenas y tribales. Cali, Colombia: Universidad Icesi.

Acemoglu, D. y Robinson, J. (2015). Por qué fracasan los países. Bogotá D. C., Colombia: Deusto.

Ballesteros, C. (2015). La mujer y el ejercicio efectivo del poder en Colombia, una cuestión de derechos humanos. Revista Verba Iuris, 33, 61-76. Documento extraído el 8 de mayo de 2018 de https://revistas.unilibre.edu.co/index.php/verbaiuris/article/view/27/24

Barragán, D. (2016). La construcción de la mentalidad democrática como necesidad en el posconflicto. Revista Via Inveniendi et Iudicandi, 11(1), 37-57.

Borrero, G. y Camilo, A. (2018). Territorialidad, organización politica y etnicidad en Colombia. Normas, jurisprudencia y categorías jurídicas aplicables a las poblaciones étnicas en Colombia. Bogotá, D. C., Colombia: Universidad Nacional de Colombia e Instituto Colombiano de Antropología e Historia.

Becerra, C. (2009). Construcción del Puerto Brisa en el territorio ancestral, destrucción del cerro sagrado Jukulwa y desconocimiento de los derechos fundamentales de los pueblos indígenas koguis, arhuacos, kankuamos y wiwas de la Sierra Nevada de Santa Marta. El derecho a la consulta previa en América Latina. Del reconocimiento formal a la exigibilidad de los derechos de los pueblos indígenas. El Otro Derecho, n. ${ }^{\circ} 40$. Instituto Latinoamericano de Servicios Legales Alternativos. Bogotá: D.C., Colombia.

Constitución Política de Colombia [C.P.]. Arts. 1º 7º, 8º 79, 246 y 330. Julio 7 de 1991. Colombia. 
Colombia. Ley 89 de 1890. Por la cual se determina la manera como deben ser gobernados los salvajes que vayan reduciéndose a la vida civilizada.

Colombia. Ley 21 de 1991. Por medios de la cual se aprueba el Convenio número 169 sobre pueblos indígenas y tribales en países independientes, adoptado por la $76^{\mathrm{a}}$ reunión de la Conferencia General de la Organización Internacional del Trabajo. Ginebra, 1989.

Colombia. Ley 165 de 1994. Por medio de la cual se aprueba el "Convenio sobre la Diversidad Biológica”. Hecho en Río de Janeiro el 5 de junio de 1992.

Colombia. Decreto-Ley 4633 de 2011. Por medio del cual se dictan medidas de asistencia, atención, reparación integral y de restitución de derechos territoriales a las víctimas pertenecientes a los pueblos y comunidades indígenas.

Ceamanos, R. (2016). El reparto de África. De la Conferencia de Berlín a los conflictos actuales. Madrid, España: Catarata y Casa África.

Coronado Conchakala, B. (2015). Kogui y wiwa en la cuenca del río Jerez. La Guajira, Colombia: Editorial Orígenes.

Consejo Territorial de Cabildos Indígenas de la Sierra Nevada de Santa Marta (2015). Documento madre de la Línea Negra —Jaba Séshizab- de los cuatro pueblos indígenas de la Sierra Nevada de Santa Marta. Santa Marta, Colombia: Cabildo Indígena Resguardo Kankuamo.

Figuera Vargas, S. C. y Sarmiento Erazo, J. P. (2016). Autodeterminación indígena en Colombia. Estudio jurídico-político del caso de la comunidad Mokaná de Malambo en el Caribe colombiano. Barranquilla, Colombia: Universidad del Norte y Grupo Editorial Ibáñez.

Garcés, A. (2016). El derecho a la consulta previa. Echando un pulso a la nación homogénea. Bogotá D. C, Colombia.: Editorial Pontificia Universidad Javeriana.

Garzón, E. (2013). Globalización del derecho, fetichismo legal el velo de los derechos humanos. Revista Verba Iuris, 30, 56-78.

Guarín, E. A. y Aldana, J. (2016). Estado jurisdiccional y bien común. Revista Verba Iuris, 11(36), 13-26.

Langford, M., Rodríguez, C. y Rossi, J. (2017). La lucha por los derechos sociales. Los fallos judiciales y la disputa politica por su cumplimiento. Bogotá D. C., Colombia: Editorial Dejusticia. 
Ministerio del Interior y de Justicia (2008). La consulta previa a pueblos indígenas y tribales. Compendio de legislación, jurisprudencia y documentos de estudio. Bogotá, D. C., Colombia: Imprenta Nacional de Colombia.

Molina González, Y. (2016). Derecho penal del enemigo y dignidad humana. Revista Verba Iuris, 11(36), 135-146.

Morales, J. (2014). La consulta previa: un derecho fundamental. Bogotá D. C., Colombia: Ediciones Doctrina y Ley Ltda., Universidad Santo Tomás y Universität Konstanz.

Murillo, E. y Duván, R. (2013). Ley de Origen y legislación en Colombia: contraposición, intereses y contradicciones entre los pueblos indígenas y el Estado, en materia de explotación de recursos naturales en la Sierra Nevada de Santa Marta. Bogotá, D. C., Colombia: Universidad Nacional de Colombia.

Natan, E. (2017). Revisión de normas internacionales del trabajo: análisis critico de las decisiones sobre temas indígenas y propuesta para una acción de la conferencia internacional del trabajo (2018).

Patarroyo, S. y Benavides, P. (2014). Rupturas asignificantes: revisiones críticas en torno al derecho. Revista Via Inveniendi et Iudicandi, 9(1), 7-31.

Rodríguez, A. C., Santamaría Chavarro, A. de. P., Acosta, M., Aparicio, M., Basset, Y., Bosa, B. ... Franco-Cuervo, A. B.(2012). Escuela Intercultural de Diplomacia Indígena. Memoria, derecho y participación. La experiencia del pueblo arbuaco, Nabusimake, Sierra Nevada de Santa Marta. Bogotá, D. C.: Universidad del Rosario.

Rodríguez, C. (2011). Etnicidad.gov. Los recursos naturales, los pueblos indígenas y el derecho a la consulta previa en los campos sociales minados. Bogotá D. C.: Centro de Estudios de Derecho, Justicia y Sociedad, Dejusticia.

Rodríguez, C. y Orduz, N. (2012). La consulta previa: dilema y soluciones. Lecciones del proceso de construcción del decreto de reparación y restitución de tierras para pueblos indígenas en Colombia. Bogotá, D. C.: Centro de Estudios de Derecho, Justicia y Sociedad. Dejusticia.

Rodríguez, G. (2017). De la consulta previa al consentimiento libre previo e informado a pueblos indígenas en Colombia. Bogotá D. C.: Universidad del Rosario y Editorial Ibáñez.

Rodríguez, G., Albán, D. y Moncayo, H. (2015). Las rutas de la consulta. Una discusión sobre la reglamentación de la consulta previa, libre e informada. Bogotá D. C.: Instituto Latinoamericano para una Sociedad y un Derecho Alternativos, Ilsa. 
Rodríguez, M. E. (2016). Estado, constitución y territorialidad. Bogotá, D. C.: Universidad Libre.

Rodríguez-Patarroyo, M. (2016). Retos jurídicos para la consulta previa a partir del análisis de su operación en el sector minero. Bogotá, D. C., Colombia: Universidad Externado de Colombia.

Saade, M. y Páramo, C. (2018). Lugares sagrados: definiciones y amenazas. Prolegómenos a la elaboración de una politica pública dirigida a los pueblos indígenas. Bogotá, D. C.: Instituto Colombiano de Antropología e Historia.

Sánchez, B., E., Jaramillo, S. y Isabel C. (2000). La jurisdicción especial indígena. Bogotá D. C.: Instituto de Estudios del Ministerio Público.

Sierra, T., Castelblanco, L., Prada, M., Salinas, Y. y Lemaitre, J. (2010). Derechos enterrados. Comunidades étnicas y campesinas en Colombia, nueve casos de estudios. Bogotá, D. C.: Universidad de los Andes, Facultad de Derecho y Centro de Investigación Sociojurí$\operatorname{dicos}$ (Cijus).

Soliz, C. (2012). El otro de América Latina. En diálogo con la emergencia indígena en América Latina, de José Bengona. México: Revista Mexicana de Política Exterior.

Vallejo, G. (2018). Convenio 169 de la Organización Internacional del Trabajo como instrumento para la protección de los territorios ancestrales de los pueblos indígenas y tribales. Bogotá D. C.: Universidad Católica de Colombia.

Víquez, S. (2019). Convenio sobre poblaciones indígenas y tribuales 1957 (núm. 107). Revista Internacional y Comparada de Relaciones Laborales y Derecho del Empleo. Escuela Internacional de Alta Formación en Relaciones Laborales y de Trabajo. 